\title{
Preparation, characterization and stability of silver sulfadiazine nanoliposomes
}

\author{
Delia Alkhatib', Noha Zelai ${ }^{2 *}$ \\ ${ }^{1}$ Department of Biochemistry, ${ }^{2}$ Department of Biological Sciences, Faculty of Science, King Abdulaziz University, Jeddah, Saudi \\ Arabia \\ *For correspondence: Email: nzelai@kau.edu.sa; Tel: +966509357153
}

\begin{abstract}
Purpose: To prepare, characterize and investigate the stability and drug release profile of silver sulfadiazine (AgSD) nanoliposomes as functions of $\mathrm{pH}, \mathrm{UV}$ exposure, and/or heat.

Methods: Silver sulfadiazine (AgSD) nanoliposomes were prepared. The stability and release properties of the free drug and nanoliposomal formulations of AgSD as functions of $\mathrm{pH}$, UV exposure, and/or heat were analyzed. The analyses were performed at different time points.

Results: The results of characterization showed that acidic, basic and neutral silver sulfadiazine nanoliposomal suspensions (AgSD NLSs) were produced as stable homogenous formulations, as indicated by low polydispersity index (PDI) and high zeta potential. The three AgSD NLSs and AgSD were unstable under different heat and/or UV conditions. The $\mathrm{pH}$ tended to shift towards neutrality, starting from day 1. Silver sulfadiazine nanoliposomal suspensions (AgSD NLSs) and free drug (AgSD) exhibited similar release properties (100\% of the drug was released at day 12).

Conclusion: Incorporation of AgSD into nanoliposomes has no significant effect on the stability and in vitro release properties of the drug. This indicates that NLS did not change the physical characteristics of the drug. Studies that focus only on improving the lipophilicity of silver sulfadiazine may benefit from these results.
\end{abstract}

Keywords: Malaria, Nanoliposomes, Drug release, Silver sulfadiazine, Suspension

\begin{abstract}
This is an Open Access article that uses a fund-ing model which does not charge readers or their institutions for access and distributed under the terms of the Creative Commons Attribution License (http://creativecommons.org/licenses/by/4.0) and the Budapest Open Access Initiative (http://www.budapestopenaccessinitiative.org/read), which permit unrestricted use, distribution, and reproduction in any medium, provided the original work is properly credited.
\end{abstract}

Tropical Journal of Pharmaceutical Research is indexed by Science Citation Index (SciSearch), Scopus, International Pharmaceutical Abstract, Chemical Abstracts, Embase, Index Copernicus, EBSCO, African Index Medicus, JournalSeek, Journal Citation Reports/Science Edition, Directory of Open Access Journals (DOAJ), African Journal Online, Bioline International, Open-J-Gate and Pharmacy Abstracts

\section{INTRODUCTION}

Malaria, a life-threatening, febrile and infectious disease which is a public health concern, affects over 5 million people in the tropics [1]. According to World Health Organization (WHO), there were an estimated 219 million cases of malaria, and 435,000 related deaths in 2017 [1]. In Saudi Arabia, approximately 177 new cases of malaria were diagnosed in 2017 , while about 2,638,680 people were at risk of the disease [1]. The surge in malaria cases in Saudi Arabia is due to the migration of refugees into the country from neighboring Yemen. Increased drug resistance is responsible for the rise in malaria cases worldwide [2]. Therefore, there is need to employ nanotechnology and nanomedicine for the development of novel antimalarials that can effectively combat resistant strains. Nanotechnology was only recently identified as 
an effective tool in drug design/delivery. The procedure increases chemical activity via enhancement of surface-to-volume ratio and reduction of the resistance of parasites via drug entrapment in a suitable delivery system $[3,4]$.

Drugs currently used for the treatment of malaria function through different mechanisms. One of such drugs is AgSD. It is a sulfonamide with silver ion $\left(\mathrm{Ag}^{+}\right)$which combines the effects of heavy metal and sulfadiazine. Originally, AgSD was used for the treatment of burn infections caused by Pseudomonas aeruginosa and other microorganisms [5]. The drug kills waterborne bacteria such as Salmonella typhi, Vibrio cholera, Staphylococcus aureus and Escherichia coli [6]. However, it produces serious side effects such as diarrhea, vomiting, nausea, hepatic necrosis, anemia and hypersensitivity reactions [7]. Although stable, AgSD is insoluble in water, alcohol and ether, and it has a low hydrophiliclipophilic balance $[8,9]$. Little or nothing is known about the stability of nanoliposome-encapsulated AgSD. The only attempt so far was the study involving chloroquine. The antimalarial drug chloroquine was loaded in liposomes to study its stability with time as functions of some physical characteristics in vivo $(\mathrm{pH}$, duration of exposure and drug concentration). The results showed that chloroquine in liposomes was more stable and less toxic than when it is in free form [10].

The aim of the present study was to prepare, characterize and investigate the stability and release profile of AgSD nanoliposomes as functions of $\mathrm{pH}$, UV exposure, and/or heat.

\section{MATERIALS AND METHODS}

\section{Materials}

All chemicals and reagents used in this study were of analytical grade. Methanol was product of Fisher chemicals (USA). Acetone was purchased from Tedia Co. Ltd. (USA). Hydrochloric acid (37 \%), monopotassium phosphate $\left(\mathrm{KH}_{2} \mathrm{PO}_{4}\right)$, acetonitrile, triethylamine and phosphoric acid were bought from SigmaAldrich (USA). Dimethyl sulfoxide (DMSO) was obtained from Loba Chemie (India). Phosphatebuffered saline (PBS) was product of Micromaster Laboratories (India).

High performance liquid chromatography (HPLC) machine was product of Shimadzu (Japan). Zetasizer was bought from Malvern Co. (UK). Sunlight meter was a product of Fabulous LLC (USA), while $\mathrm{pH}$ meter was purchased from Jenway Co. Ltd. (UK).
Preparation of AgSD nanoliposomes and drug loading

In one beaker, cationic nanoliposomes were prepared by dissolving $50 \mathrm{mg}$ of dimethyl dioctadecyl ammonium bromide (DDA) in $50 \mathrm{~mL}$ of chloroform. In another beaker, $50 \mathrm{mg}$ of cholesterol was dissolved in $50 \mathrm{~mL}$ of chloroform. A third beaker was used to dissolve $50 \mathrm{~mL}$ of AgSD in $50 \mathrm{~mL}$ of mixed solvents of $33 \%$ ammonium hydroxide and water $(1: 10, v / v)$. The contents of the three beakers were then mixed in volume ratio of 2:1.5:0.5 (DDA: AgSD: cholesterol). After vortexing, the mixture was transferred to a small glass vial and dried using stream of nitrogen gas. The glass vial was left under vacuum for $1 \mathrm{~h}$ to obtain a dry lipid film which was rehydrated with normal saline and heated for $15 \mathrm{~min}$ at $60{ }^{\circ} \mathrm{C}$. Subsequently, the content of the vial was transferred to an Eppendorf tube and centrifuged at 10,000 rpm for $5 \mathrm{~min}$ to precipitate both non-encapsulated drug and non-hydrated lipids [11].

\section{Determination of AgSD content of nanoliposomal suspensions}

The drug content of the three nanoliposomal suspensions (NLSs) were analyzed with HPLC [12]. The HPLC machine was operated at 254 $\mathrm{nm}$ using C18 column and mobile phase composed of water, acetonitrile and phosphoric acid in volume ratio of 900:99:1. Exactly $0.9 \mathrm{~mL}$ of nano-formulation was drawn from each vial and added to $0.1 \mathrm{~mL}$ of the internal standard (sulfamerazine).

\section{Characterization of NLSs}

Drug characteristics such as polydispersity index (PDI), zeta potential, and size distribution were analyzed. Samples were diluted five times, and analyzed using Zetasizer.

\section{Stability studies}

Silver sulfadiazine (AgSD) and its nanoformulations were dispensed in three beakers and treated with $\mathrm{HCl}$ or $\mathrm{NaOH}$ to produce acidic (pH 4.5), basic (pH 10.5) and neutral suspensions $(\mathrm{pH} 7.4)$. These formulations were kept under different thermal and/or UV conditions. The stability of the AgSD formulations with time at the various $\mathrm{pH}$ values was determined.

\section{Stability under thermal and/or UV conditions}

Aliquots of acidic, basic and neutral AgSD NLS and free AgSD were dispensed in three beakers. 
The first beaker was kept outdoors under direct sunlight at $40-45^{\circ} \mathrm{C}$ and average UV index of $12 \mathrm{SU}$, as measured using sunlight meter (solar UV photometer). The second beaker was kept at a temperature of $24{ }^{\circ} \mathrm{C}$ and exposed to sun UV rays through window glass. The third beaker was kept outdoors under shade (heat only). Samples were drawn for analysis at different time points: $2,6,8,10,12$, and $24 \mathrm{~h}$; and on days $6,12,18$, 24 , and 30 . The samples were analyzed at 254 $\mathrm{nm}$ using HPLC, with injection volume of $10 \mu \mathrm{L}$, flow rate of $2 \mathrm{ml} / \mathrm{min}$ and $3.9 \mathrm{~mm} \times 30 \mathrm{~cm}$ column.

\section{Evaluation of the stability of the pH of AgSD and AgSD NLS}

The stability of the $\mathrm{pH}$ of AgSD and AgSD NLSs on exposure to different thermal and/or UV conditions was determined with $\mathrm{pH}$ meter. The $\mathrm{pH}$ of acidic $(\mathrm{pH} 4.5)$, basic $(\mathrm{pH} 10.5)$ and neutral $(\mathrm{pH} 7.4) \mathrm{AgSD}$ and NLSs were measured on days 1,12 and 30 to check for any changes.

\section{Drug release profiles}

The dissolutions of AgSD NLSs and AgSD at $\mathrm{pH}$ 7.4 were measured. The samples were resuspended in $200 \mathrm{~mL}$ PBS $(\mathrm{pH}$ 7.4) and incubated at $37{ }^{\circ} \mathrm{C}$. Cellophane dialysis membrane tubing (dialysis bag) containing drug formulations was inserted in each beaker. Then, aliquots of eluted drug medium were removed for analysis at different time points: at onset, $2 \mathrm{~h}, 6$ h, 8 h, 10 h, 12 h, 24 h, day 6 , day 12, day 18, day 24 , and day 30 . Each sample of PBS (0.9 $\mathrm{mL}$ ) was subjected to HPLC analysis. This volume was replaced with fresh buffer to prevent sink conditions. To each vial, $0.1 \mathrm{~mL}$ of the internal standard sulfamerazine was added [12].

\section{Statistical analysis}

Data are expressed as mean \pm standard error of mean (SEM). Groups were compared using Student $t$-test. Statistical analysis was performed with GraphPad prism version 7. Values of $p<$ 0.05 were taken as indicative of statistically significant differences.

\section{RESULTS}

\section{Characteristics of silver sulfadiazine nanoliposomes}

The zeta potential of AgSD NLSs were significantly higher than that of blank NLS ( $p<$ $0.05)$, but there were no significant differences in zeta potential among the three nanoliposomal suspensions $(p>0.05)$. Moreover, there were no significant differences in zeta sizes at all $\mathrm{pH}$ conditions among the groups $(p>0.05)$. However, PDI was significantly higher in blank NLS than in AgSD NLSs $(p<0.05$; Table 1).

\section{Drug stability under different heat and/or UV conditions}

The three formula groups: acidic, basic and neutral AgSD NLSs and AgSD were kept under direct sunlight, shadow and sunlight without heat. Samples were taken at different time points ranging from zero time (immediately) to the $30^{\text {th }}$ day. The results showed that formulations were not stable under these conditions, but the degree of instability varied among the formulas $(p<$ 0.05). Under "no heat" condition (exposure of formulations to UV light), there was significant and time-dependent difference in drug stability between acidic and neutral AgSD NLSs ( $p<$ 0.05 ). The basic AgSD NLS was stable only for 2 $h$, beyond which it was degraded. Although acidic AgSD was stable for $2 \mathrm{~h}$, the concentrations of the other AgSD at all $\mathrm{pH}$ conditions were unstable. However, under shadow conditions (heat, without UV light), the acidic AgSD NLS was stable for 6 , beyond which the drug content fluctuated and returned to normal on day 30 ( $p=0.9688)$. Neutral AgSD was stable for $2 \mathrm{~h}$. All other formulas showed instability with time under shadow $(p<0.05)$. In solar conditions (exposure to UV light and heat), all the formulas (acidic, basic and neutral NLSs and AgSD) were unstable $(p<0.05)$. Moreover, NLS formulations were completely degraded at day 24; acidic and neutral AgSD samples were completely degraded at day 18 , while basic AgSD was degraded at day 30 . These results are shown in Figure 1, Figure 2 and Figure 3.

Table 1: Physical properties of AgSD NLSs and blank

\begin{tabular}{lccc}
\hline Group & Zeta potential $(\mathbf{m V})$ & Average size $(\mathbf{n m})$ & PDI \\
\hline Acidic AgSD NLS (pH 4.5) & $36.90 \pm 1.80^{\mathrm{a}}$ & $354.70 \pm 5.70$ & $0.37 \pm 0.01^{\mathrm{a}}$ \\
Neutral AgSD NLS (pH 7.4) & $37.70 \pm 6.50^{\mathrm{a}}$ & $455.50 \pm 54.10$ & $0.48 \pm 0.03^{\mathrm{a}}$ \\
Basic AgSD NLS (pH 10.5) & $34.20 \pm 4.60^{\mathrm{a}}$ & $335.40 \pm 8.30$ & $0.49 \pm 0.04^{\mathrm{a}}$ \\
Blank NLS & $-2.70 \pm 0.09$ & $409.70 \pm 9.40$ & $0.73 \pm 0.05$ \\
\hline a $>$ 0.05 compared with blank NLS; AgSD NLS = silver sulfadiazine nanoliposomal suspension; $\mathrm{mV}=$ millivolt; \\
nm = nanometer; and PDI = polydispersity index
\end{tabular}


A

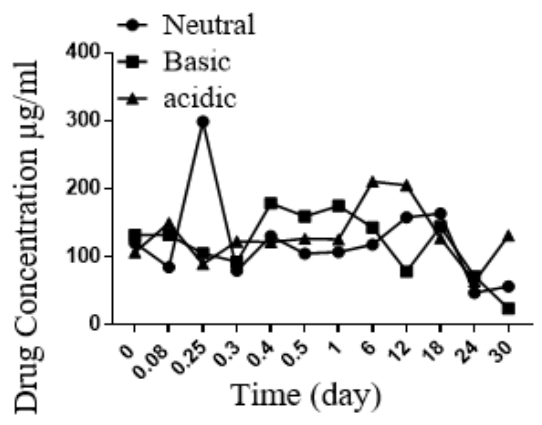

B

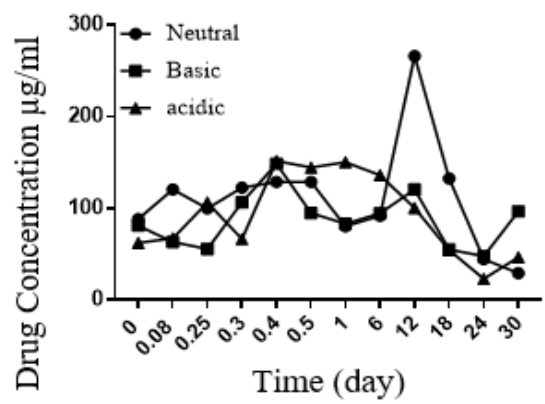

Figure 1: Stability of AgSD NLSs and AgSD under "no heat" condition. (A) AgSD NLS in UV exposure condition. (B) AgSD in UV exposure condition

A

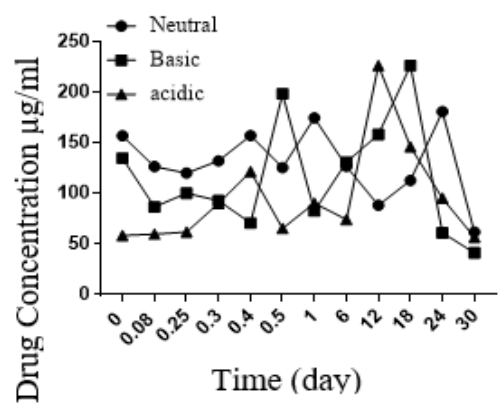

B

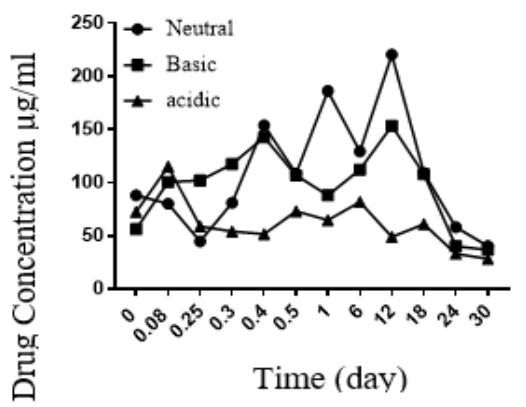

Figure 2: Stability of AgSD NLSs and AgSD under shadow conditions. (A) AgSD NLS in heat condition. (B) AgSD in heat conditions
A

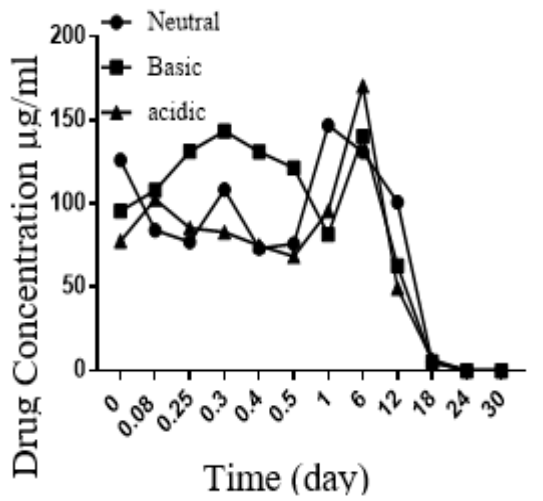

B

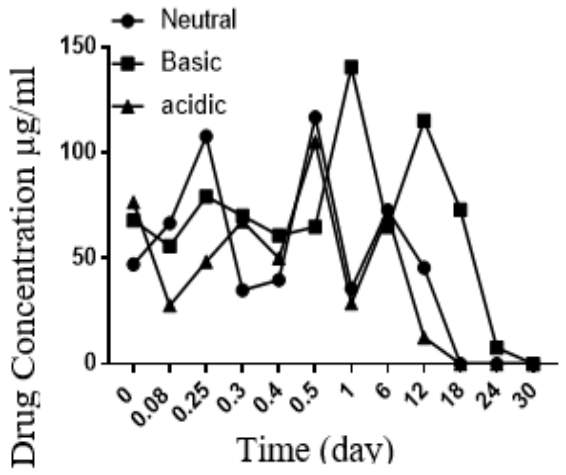

Figure 3: Stability of AgSD NLSs and AgSD under solar conditions. (A) AgSD NLS in UV exposure and heat conditions. (B) AgSD in UV exposure and heat conditions

\section{Effect of heat and/or UV on the stability of $\mathrm{pH}$ of AgSD NLS and AgSD formulations}

The stability of $\mathrm{pH}$ of AgSD NLSs and AgSD were monitored for a period of 1 month. As shown in Figure 4, the $\mathrm{pH}$ of the acidic and basic AgSD NLSs and AgSD was approximately 7. Although the $\mathrm{pH}$ of most AgSD NLSs rose to approximately 9 on day 30 , the change in $\mathrm{pH}$ was not significantly different on exposure to sunlight without heat, shadow and solar conditions $(p>0.05)$. The $\mathrm{pH}$ shifted toward neutrality with time.

\section{Drug release}

Nanoliposomal suspensions (NLSs) of AgSD did not release drug on day 1 . The results showed that $100 \%$ of the drug was released at day 12 . However, there were no significant differences in drug concentrations among all the formulations on days 6 and 12 ( $p>0.05$; Figure 5). 
(A) $\mathrm{pH}$ under UV exposure conditions

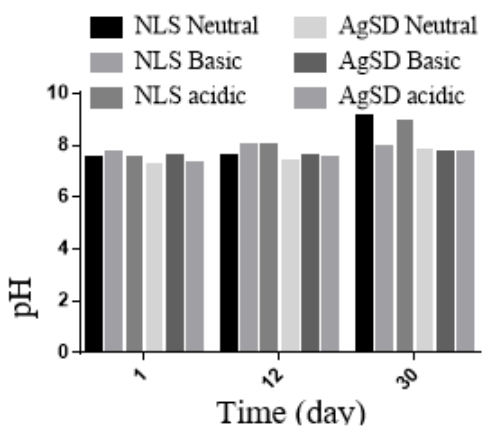

(B) $\mathrm{pH}$ under heat conditions

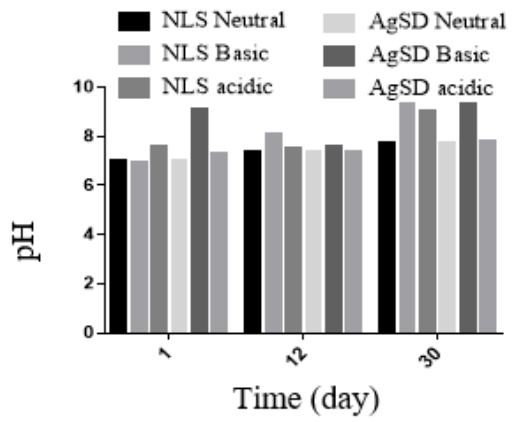

(C) $\mathrm{pH}$ under UV exposure and heat conditions

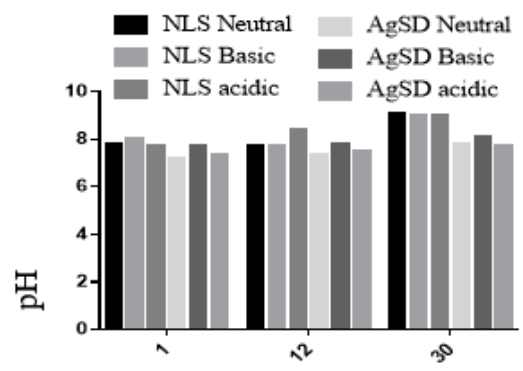

Time (day)

Figure 4: Heat and/or UV effect on the stability of $\mathrm{pH}$ of AgSD NLS and AgSD formulations. (A): Under UV exposure; (B): Under shadow condition; and (C): Under heat condition

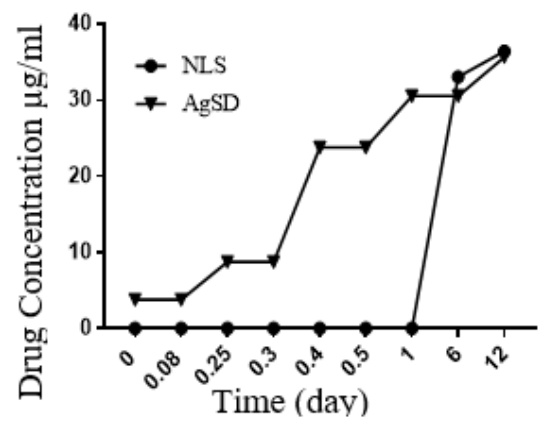

Figure 5: Drug release profile

\section{DISCUSSION}

Malaria, a disease caused by infection by a single-cell parasite Plasmodium, remains one of the leading causes of morbidity and mortality in the tropics. About 655,000 persons died of malaria in 2010 and $86 \%$ of the victims were children under 5 years of age [1]. Antimalarial drugs are used in three different ways: prophylaxis, treatment of falciparum malaria and treatment of non-falciparum malaria. Treatment protocols for falciparum malaria vary, depending on severity of the disease: fast-acting, parenteral drugs are best suited for severe, life-threatening disease [1]. Drugs currently used for the treatment of malaria employ various mechanisms. One of such drugs is AgSD [5]. The aim of this study was to prepare, characterize and investigate the stability and release profile of AgSD nanoliposomes as functions of $\mathrm{pH}, \mathrm{UV}$ exposure, and/or heat. The results of characterization showed that acidic, basic and neutral AgSD NLSs produced stable homogenous formulations, as indicated by low PDI and high zeta potential.

Size, distribution and physical integrity are parameters commonly used to characterize and evaluate the quality of NLS $[13,14]$. Hydrochloric acid $(\mathrm{HCl})$ which was used to adjust the $\mathrm{pH}$ of AgSD NLSs ( $\mathrm{pH}$ of the basic NLS was adjusted with $\mathrm{NaOH}$ ), may have increased the positive charge, thereby influencing the zeta potential. Similarly, the silver ion $\left(\mathrm{Ag}^{+}\right)$may have influenced the positive charge on the formulations. The low zeta potential and high PDI of blank NLS indicate heterogenicity [15].

Nanoliposomes were used in this study, since they have been successfully employed as biopreservatives to entrap food, vitamin $D$, minerals (such as $\mathrm{Ca}^{2+}$ and $\mathrm{Mg}^{2+}$ ), pharmaceuticals and cosmetics, thereby protecting them from degradation [16-20]. In this study, both AgSD NLSs and AgSD were unstable under different heat and/or UV conditions. These results suggest that the formulations should be kept away from heat and UV light. Similar results have been obtained in previous reports [21]. Studies have shown that nanoliposome-entrapped drugs are readily and rapidly degraded when exposed to increased heat (in both UV and dark conditions) [22-24].

Measurement of $\mathrm{pH}$ at different time points showed that it tended to shift towards neutrality, starting from day 1 . It is likely that the $\mathrm{pH}$ of water (6.5 - 8.5) and AgSD (4 - 6) overwhelmed the effect of $\mathrm{HCl}$ and $\mathrm{NaOH}$ [21]. In a previous study, the $\mathrm{pH}$ of chloroquine liposome decreased 
with time, and it was attributed to bacterial contamination [23]. The results of previous studies have also demonstrated that abrupt change in $\mathrm{pH}$ can be detrimental to the structure of liposomes [25].

Moreover, the release profile of AgSD NLSs and AgSD revealed that both were released over the same period of time. The lag phase of AgSD NLSs may be due to aggregation of NLS in the pores of the dialysis bag, which was not the case with AgSD solution [26]. The release of $91 \%$ of the drug at day 6 indicated that most of the AgSD NLSs were released between days 1 and 6 . The results of a previous study showed that nanoliposomes with $30 \%$ cholesterol exhibited greater stability, as well as controllable and reproducible release. This may suggest that increasing cholesterol ratio from 0.5 to 1 could improve the drug release profile.

\section{CONCLUSION}

Incorporation of AgSD into nanoliposomes has no significant effect on the stability and in vitro release properties of the drug. This indicates that NLS does not change the physical characteristics of the drug. Studies that focus only on improving the lipophilicity of silver sulfadiazine may benefit from these finding.

\section{DECLARATIONS}

\section{Acknowledgement}

Special thanks go to the Science Research and Innovation Unit and Dr Shareefa Alghamdi of Faculty of Science, King Abdulaziz University and Mawakeb Alajer for supporting this work.

\section{Conflict of interest}

No conflict of interest is associated with this work.

\section{Contribution of authors}

All authors conceptualized and designed the experiments. Delia Alkhatib conducted most of the literature search and experiments and wrote the first draft of the manuscript. Noha Zelai performed the statistical analysis and edited the manuscript.

\section{Open Access}

This is an Open Access article that uses a funding model which does not charge readers or their institutions for access and distributed under the terms of the Creative Commons Attribution License (http://creativecommons.org/licenses/by/ 4.0) and the Budapest Open Access Initiative (http://www.budapestopenaccessinitiative.org/rea d), which permit unrestricted use, distribution, and reproduction in any medium, provided the original work is properly credited.

\section{REFERENCES}

1. World Health Organization. World Malaria Report, 2018 [cited 2020 November 19].

2. Harvey A, Howland D, Champe C, Mycek J. Lippincott's illustrated reviews: Pharmacology. Lippincott Williams \& Wilkins Philadelphia, 6th edn. Florida: Wolters Kluwar; 2014.

3. Morones R, Elechiguerra L, Camacho A, Holt K, Kouri B, Ramírez $T$, Yacaman MJ. The bactericidal effect of silver nanoparticles. J Nanotechnol. 2005; 16(10): 23462353.

4. Rajendran V, Rohra S, Raza M, Hasan GM, Dutt S, Ghosh PC. Stearylamine Liposomal Delivery of Monensin in Combination with Free Artemisinin Eliminates Blood Stages of Plasmodium falciparum in Culture and $P$. berghei Infection in Murine Malaria. Antimicrob Agents Chemother. 2015; 60 (3): 1304-1318.

5. Thurston P. Morphological changes in Plasmodium berghei following proguanil, sulphadiazine and mepacrine therapy. Trans $R$ Soc Trop Med Hyg, 1951; 44: 703-706

6. Jaganathan $A$, Murugan $K$, Panneerselvam $C$, Madhiyazhagan $P$, Dinesh $D$, Vadivalagan $C$, Aziz A, Chandramohan $B$, Suresh $U$, Rajaganesh $R$ et al. Earthworm-mediated synthesis of silver nanoparticles: $A$ potent tool against hepatocellular carcinoma, Plasmodium falciparum parasites and malaria mosquitoes. Parasitol Int. 2016; 65 (3): 276-284.

7. Torok E, Moran E, Cooke F. Oxford Handbook of Infectious Diseases and Microbiology. 2nd. Oxford, UK: Oxford Medical Handbooks; 2009.

8. Ballin C. Evaluation of a New Topical Agent for Burn Therapy: Silver Sulfadiazine (Silvadene). JAMA. 1974; 230 (8): 1184-1185.

9. Nesbitt U, Sandmann J. Solubility Studies of Silver Sulfadiazine. J. Pharm. Sci. 1977; 66 (4): 519-522,

10. Nieuwoudt S. Preparation, stability and in vitro evaluation of liposomes containing chloroquine. North-West University. 2010.

11. Benatti R, Epand M, Lamy T. Low cholesterol solubility in dodab liposomes. Chem Phys Lipids. 2006; 145 (1): 2736.

12. The United States Pharmacopeia and the National Formulary (USP). 1st Ed. New York, United States: Pharmacopeial 2016.

13. Samuni AM, Barenholz Y. In: Liposomes, Methods in Enzymology; Düzgüne $N$, editor. Elsevier Academic Press: USA, 2004; pp. 299-314.

Trop J Pharm Res, April 2021; 20(4): 670 
14. Castor TP, Chu L. Methods and apparatus for making liposomes containing hydrophobic drugs. US5776486, 1998.

15. Patravale V, Dandekar $P$, Jain R. 3-Characterization techniques for nanoparticulate carriers. In: Patravale $V$, Dandekar $P$, Jain $R$, editors. Nanoparticulate Drug Delivery: Woodhead Publishing 2012; pp. 87-121.

16. Mozafari MR, Flanagan J, Matia-Merino L, Awati A, Omri $A$, Suntres ZE, Singh $H$. Recent trends in the lipid-based nanoencapsulation of antioxidants and their role in foods. J. Sci. Food Agric. 2006; 86 (13): 2038-2045.

17. Garti N. Delivery and controlled release of bioactives in foods and nutraceuticals; Woodhead Publishing Limited: USA, 2008.

18. Acosta E. Testing the effectiveness of nutrient delivery systems. In: Delivery and controlled release of bioactives in foods and nutraceuticals; Garti, N, Ed.; Woodhead Publishing Limited: England, 2008; pp 53-97.

19. Mozafari MR. Nanoliposomes: preparation and analysis. Methods Mol Biol. 2010; 605: 29-35.

20. Silva-Malheiros $P$, Daroit DJ, Brandelli A. Food applications of liposome-encapsulated antimicrobial peptides. Trend Food Sci. Tech. 2010; 21 (6): 284-292.
21. Plumb C. Plumb's veterinary drug handbook. 8th Ed. Wiley-Blackwell 2015. Pp. 1801-1802.

22. Ko S, Lee SC. Effect of nanoliposomes on the stabilization of incorporated retinol. Afr J Biotechnol. 2013; 9 (37): 6158-6161.

23. Barattin M, Mattarei A, Balasso A, Paradisi C, Cantù L, Del-Favero E, Viitala T, Mastrotto F, Caliceti P, Salmaso S. pH-Controlled Liposomes for Enhanced Cell Penetration in Tumor Environment. ACS Appl Mater Interfaces. 2018; 10 (21): 17646-17661.

24. Taira MC, Chiaramoni NS, Pecuch KM, AlonsoRomanowski S. Stability of liposomal formulations in physiological conditions for oral drug delivery. Drug Deliv. 2004; 11 (2): 123-128.

25. Khosravi-Darani K, Mozafari MR. Nanoliposome potentials in nanotherapy: A concise overview. J. Nanosci. Nanotechnol. 2010; 6: 3-13.

26. Souza S. A Review of In Vitro Drug Release Test Methods for Nano-Sized Dosage Forms. Adv Pharm. 2014; 1-12.

27. Briuglia ML, Rotella C, McFarlane A, Lamprou DA. Influence of cholesterol on liposome stability and on in vitro drug release. Drug Deliv Transl Res. 2015; 5 (3): 231-242. 